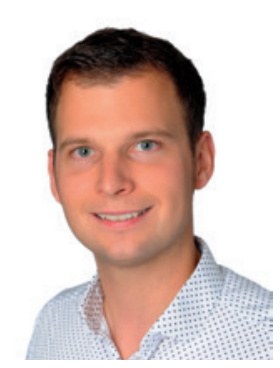

\title{
Automatisierte Diagnosestellung und Vermessung des Strabismus bei Kindern
}

\author{
Sebastian Siebelmann
}

Augenklinik, Uniklinik Köln, Köln, Deutschland

Abstract aus Yehezkel O, Belkin M, Wygnanski-Jaffe T: Automated Diagnosis and Measurement of Strabismus in Children. Am J Ophthalmol 2020;213:226-234.

\begin{abstract}
Purpose: Manual measurements of strabismus are subjective, time consuming, difficult to perform in babies, toddlers, and young children, and rely on the examiner's skill and experience. An automated system, based on eye tracking and dedicated full occlusion glasses, was developed to provide a fast, objective, and easy-to-use alternative to the manual measurements of strabismus. This study tested the efficacy of the system in determining the presence of strabismus in children, as well as its type and the amount of deviation, in addition to differentiating between phorias and tropias.
\end{abstract}

Design: A prospective, masked, inter-rater reliability study. Methods: A prospective, masked, cross-sectional study included 69 children, 3-15 years of age. A cover-uncover test and a prism alternating cover test (PACT) for the primary gaze, at a distance of
$50 \mathrm{~cm}$, were performed by 2 independent, masked examiners and by the automated system.

Results: A high correlation was found between the automated and the manual test results $(R=0.9$ and $P<0.001$ for the horizontal deviation, and $R=0.91$ and $P<0.001$ for the vertical deviations, with $100 \%$ correct identification of the type of deviation). The average automated test duration was 46 seconds. The Bland-Altman plot, used to compare the 2 measurement methods, showed a mean value of -2.9 prism diopters (PD) and a half-width of the 95\% limit of agreement of \pm 11.4 PD.

Conclusion: The automated system provides precise detection and measurements of ocular misalignment and differentiated between phorias and tropias. It can be used in participants from the ages of 3 years old and up.

(c) 2019 The Author(s). 


\section{Transfer in die Praxis}

\section{Hintergrund}

Die klinische Untersuchung von Kleinkindern ist aufgrund einer meist nur eingeschränkten Kooperationsfähigkeit eine Herausforderung. Meist erfordert die korrekte Diagnosestellung nicht nur ausreichend Zeit, sondern auch eine langjährige Erfahrung der Untersucher. Beides ist im klinischen Alltag nur begrenzt verfügbar, sodass elektronische, automatisierte Hilfssysteme zur Diagnosestellung beim Strabismus eine deutliche Erleichterung darstellen könnten. In der hier vorliegenden prospektiven, verblindeten Studie wurde ein automatisches System evaluiert, das mithilfe einer Eye Tracking-Technik nicht nur das Vorliegen eines Strabismus bei Kindern detektiert, sondern auch die genaue Art des Strabismus sowie dessen Grad der Abweichung bestimmen kann. Zudem kann das verwendete System zwischen Phorien und Tropien unterscheiden. Als Referenz dienten dabei 2 unabhängige, verblindete Untersucher.

\section{Eye Tracking}

Als Eye Tracking wird die Erfassung von Blickbewegungen durch sogenannte Eyetracker bezeichnet. Eyetracker zeichnen Blickbewegungen auf und analysieren diese. Erstaunlicherweise wird Eye Tracking nicht nur in der Augenheilkunde genutzt, sondern auch in den Neurowissenschaften. Einen besonderen Stellenwert nimmt das Eye Tracking für die Werbeindustrie ein. Unter Anwendung von z.B. Wahrnehmungs- und kognitionspsychologischen Methoden wird hierbei unter anderem die Anwendungsfreundlichkeit bzw. das Design von Gegenständen, Software, Websites oder Werbung analysiert und optimiert. Doch schon im 19. Jahrhundert wurden Augenbewegungen z.B. von Javal [1] manuell aufgezeichnet und analysiert. Die heutigen elektronischen Eyetracker nutzen dahingegen meist eine elektronische und Video-gestützte Technik. Generell unterscheidet man zwischen mobilen und immobilen externen Eyetrackern. Mobile Eyetracker werden meist auf den Kopf des Probanden installiert, während externe Geräte zum Teil in die jeweiligen Geräte eingebaut sind. Als Technologie, um Augenbewegungen zu tracken, eignen sich generell retinale Nachbilder, das Elektrookulogramm, spezielle Eye Tracking-Kontaktlinsen sowie die sogenannte Cornea-Reflex-Methode (video-based eye tracking). Die Cornea-Reflex-Methode, bei der der Hornhautreflex mittels einer Infrarotkamera oder einem Laser verfolgt wird, ist eine der am weitesten verbreiteten Technologien. Inzwischen sind eine Vielzahl von Geräten dazu in der Lage, ein Eye Tracking durchzuführen, darunter in Smartphones zur Nutzungsanalyse, in Kraftfahrzeugen zur Müdigkeitserkennung und nicht zuletzt in Excimerlasern zur Kontrolle der Augenposition bei der photorefraktiven Keratektomie (PRK) oder der Laser-assistierten In-situ-Keratomileusis (LASIK). In der hier vorgestellten Studie wurde ein Video- bzw. Infrarotlicht-basierter Eyetracker (Eyetracke 4C, Mississauga, Ontario, Canada) genutzt.

\section{Ergebnisse der Studie}

Die Ergebnisse der Studie konnten zeigen, dass eine hohe Korrelation zwischen den Ergebnissen des automatisierten Systems und den Ergebnissen der beiden Untersucher bestand. Sowohl horizontale, als auch vertikale Deviationen konnten zu 100\% korrekt erkannt werden. Als Dauer des entsprechenden Tests waren bis zur Erkennung des Strabismus im Durchschnitt 46 Sekunden angegeben. Dabei konnte quantitativ eine Korrelation zwischen den Ergebnissen der automatischen und der manuellen Untersuchungen für Esodeviationen von 0,9, für Exodeviationen von 0,88 und für vertikale Deviationen von 0,91 erreicht werden. Zudem wurden Kinder, bei denen eine Orthophorie vorlag, korrekt als solche identifiziert. Allerdings konnten in Einzelfällen versteckte Bewegungsstörungen, wie z.B beim Brown-Syndrom, nur von den erfahrenen Untersuchern aufgedeckt werden.

\section{Fazit für die Praxis}

Automatische Diagnosesysteme zur Erkennung und Quantifizierung eines Strabismus könnten in Zukunft die Untersuchung von Kindern deutlich erleichtern. Zudem könnte diese Art der Diagnostik in weniger gut mit erfahrenen Untersuchern versorgten Gebieten einen Beitrag zur Früherkennung und Amblyopieprophylaxe von Kleinkindern leisten. Dennoch konnte in der Studie ebenfalls festgestellt werden, dass komplexe Bewegungsstörungen oftmals nur von erfahrenen Untersuchern provoziert werden konnten.

\section{Disclosure Statement}

Der Autor hat keine finanziellen Interessen.

\section{Literatur}

1 Javal E: Essai sur la physiologie de la lecture. Ann Ocul (Paris) 1878;80:240274.

Kontaktadresse: PD Dr. Sebastian Siebelmann, Augenklinik, Uniklinik Köln, Kerpener Straße 62, 50931 Köln, Deutschland, sebastian.siebelmann@ uk-koeln.de 